\title{
Re-engineering of Backoffice duties at Family Health Bureau, Sri Lanka
}

\author{
Karunatilaka M. N. ${ }^{1}$, De Silva, C. ${ }^{2}$ \\ ${ }^{1}$ Registrar in Medical Administration, ${ }^{2}$ Director, Family Health Bureau \\ DOI: 10.29322/IJSRP.11.07.2021.p11569 \\ http://dx.doi.org/10.29322/IJSRP.11.07.2021.p11569
}

\begin{abstract}
The objective of this research was to evaluate the back-office functions at the Family Health Bureau (FHB) for the purpose of efficient reallocation of duties to achieve better output through the approved carder and descriptive cross-sectional study design was used.

Approved cadre for the office was only 11 Public Management Assistants (PMA) [2] and at the study period there were 12 PMAs including Chief Clerk. There were 5 Development Officers without carder positions. [3, 4, 5].

Out of the 15 analyzed officers 2 were awaiting transfers without duty list and one officer totally idling. There were two officers with less than average 5-day work per month and eight offices with less than average 15-day work. Storekeeper and Shroff was expected full time availability and total tender responsibility given to one officer. There for only $57 \%$ of available time utilized by the institution. Proposed system has only 10 subjects excluding chief clerk and duties were distributed scientifically to utilize all the employees equally and efficiently. Ten subjects were regrouped to 2 divisions under Accountant and Administrative Officer and increases the utilization of time to $79 \%$ within the approval carder. Still there is $21 \%$ of man time waste and more room to further streamline.
\end{abstract}

\section{Key words - Back office, Duty allocation, Utilization}

\section{INTRODUCTION}

Family Health Bureau (FHB) is the focal point for Maternal and Child Health (MCH) in Sri Lanka. Its vision is "A Sri Lankan nation that has optimized the quality of life and health potential of all women, children and their families". The mission of the FHB is "To contribute to the attainment of highest possible levels of health of all women, children and families through provision of comprehensive, sustainable, equitable and quality Maternal and Child Health services in a supportive, culturally acceptable and family friendly settings" [1].

The technical functions of the FHB are led and directed by the Director / FHB. Director was supported by the back office, which is comprised of accounts section and administrative section. Accountant and Administrative Officer to supervise their functions [2].

The objective of the present study is to evaluate the back-office duties at Family Health Bureau for the purpose of efficient reallocation to achieve better output through the approved carder.

\section{METHODS}


The study was carried out using two main research methods: 1) Key Informant Interviews; 2) Desk Evaluations. There were three steps in the study: 1) Search of carder information, present duties, and its distribution; 2) Interviews with 15 Public Management Assistants / Development Officers; 3) Interviews with Administration Officer, Accountant and Chief Clerk. Director's personal assistant excluded from study as she is dedicated for the director's officer work.

In the first step studied the carder and the available present duty lists. Duties of chief clerk was not analyzed as she is playing an overall supervisory job and director's personnel assistant was too not analyzed as she is dedicated for the director's office duties. The duty lists were cross check with the chief clerk on actual distribution of the duties. All 15 duty lists were revised following deleting not relevant duties and adding newly introduced duties. In the second step revised duty lists were discussed with all 15 Public Management Assistants / Development Officers with regard to time, responsibility, and availability. Estimated working days for a month was 22 days excluding weekends and holidays. Time was calculated on daily basis and multiplied by 22 to assess the monthly requirement for each duty. At the same time interviewed their perception on other's duties and coordination between peers. All the subject work was Cross examined with same type external institution in the third step. In the fourth step duty re allocation was done considering government circular to limit office duties only to the Public Management Assistants as well. In the final step revised duty lists were validated with the concurrences of Deputy Director, Accountant and Administrative Officer of the institution.

\section{RESULTS \& DISCUSSION}

Approved cadre for the office is only 11 Public Management Assistants and at present there are 12 Public Management Assistants including Chief Clerk. There is no Development Officer cadre for the office but there are five development officers in the office. There is no clear accounts division and administrative division in the office. The present duty lists analysis based on time, responsibility, and availability as follow.

\begin{tabular}{|c|l|c|}
\hline Employee No & Duty & Days \\
\hline 01 & Salary preparation \& issuing pay slips & 9 \\
\hline & Salary arrears \& Ledger balancing & 2 \\
\hline & Issuing T-10 & 1 \\
\hline & Carder report & 1 \\
\hline & & $\mathbf{1 3}$ \\
\hline
\end{tabular}

\begin{tabular}{|c|l|c|}
\hline Employee No & Duty & Days \\
\hline 02 & Overtime (Except Doctors) & 4 \\
\hline & Travelling claims (All staff) & 7 \\
\hline & & $\mathbf{1 1}$ \\
\hline
\end{tabular}

\begin{tabular}{|c|l|c|}
\hline Employee No & Duty & Days \\
\hline 03 & Leave, Train pass, Train warrant & 1.5 \\
\hline & Overtime \& travelling voucher checking & 3 \\
\hline & Petty cash balance (School Health Office) & 0.5 \\
\hline & Casual employee attendance verification for salary & 1 \\
\hline
\end{tabular}

This publication is licensed under Creative Commons Attribution CC BY. 


\begin{tabular}{|c|l|c|}
\hline Employee No & Duty & Days \\
\hline 04 & Vote ledger \& reports & 4 \\
\hline & General deposit account & 1 \\
\hline & Doctors overtime \& Combined Allowance voucher & 6 \\
\hline
\end{tabular}

\begin{tabular}{|c|c|c|}
\hline Employee No & Duty & Responsibility \\
\hline \multirow[t]{4}{*}{05} & Annual procurement plane & \multirow{4}{*}{$\begin{array}{c}\text { Total tender subject } \\
\text { responsibility }\end{array}$} \\
\hline & Monthly progress of procurement & \\
\hline & Calling for tenders & \\
\hline & Calling for quotations & \\
\hline Employee No & Duty & Days \\
\hline \multirow[t]{6}{*}{06} & Cask book maintenance & 6 \\
\hline & Management of allocations (Local \& Foreign) & 3.5 \\
\hline & Purchasing of office newspaper & 0.5 \\
\hline & Allocation of foreign funds & 2 \\
\hline & Bank reconciliation & 2 \\
\hline & & 14 \\
\hline
\end{tabular}

\begin{tabular}{|c|l|c|}
\hline Employee No & Duty & Days \\
\hline 07 & Transport subject (Insurance, Repair, Daily records) & 4 \\
\hline & Vehicle fuel supply management & 3 \\
\hline & Preliminary examination of accidents & 0.5 \\
\hline & Maintenance of files on accidents & 1 \\
\hline & General, electrical, photocopy \& AC maintenance & 8 \\
\hline & & $\mathbf{1 6 . 5}$ \\
\hline
\end{tabular}

\begin{tabular}{|c|l|c|}
\hline Employee No & Duty & Availability \\
\hline 08 & Proper maintenance of the stores & \multirow{2}{*}{ Full time stores } \\
\hline & Item estimation \& requesting & \\
\hline & Maintenance of record books & \\
\hline & Issue of office items & \\
\hline & Issue items for janitorial service & \\
\hline
\end{tabular}




\begin{tabular}{|c|l|c|c|}
\hline Employee No & Duty & Time & Availability \\
\hline 09 & Shroff & & Full time \\
\hline & Petty cash book & 1 & \\
\hline & Housing loan \& 10-month loan account & $\mathbf{2}$ & \\
\hline & & & \\
\hline
\end{tabular}

\begin{tabular}{|c|l|c|}
\hline Employee No & Duty & Days \\
\hline 10 & Laundry bill settlement & \multirow{2}{*}{1} \\
\hline & Office tax payments & \\
\hline & Uniform duty & 0.5 \\
\hline & Office identity card related duties & 0.5 \\
\hline & "Agrahara" claims & 1 \\
\hline & Officer allocation for mobile clinics & 1 \\
\hline & Security officers' payments & $\mathbf{0 4}$ \\
\hline & Janitorial service payments & 1 \\
\hline & &
\end{tabular}

\begin{tabular}{|c|l|c|}
\hline Employee No & Duty & Days \\
\hline 11 & Preparation of annual estimates (Once a Year) & $(22)$ \\
\hline & Preparation of expenditure reports (monthly) & 1 \\
\hline & Preparation of imprest (Liquid Cash) applications & 1 \\
\hline & Maintenance of government salary structure & 1 \\
\hline & Procurement estimates & 2 \\
\hline & Internal \& General audit reports & 5 \\
\hline & CIGAS/GPS & 4 \\
\hline & Surgical store document management & 1 \\
\hline & Preparation of appropriation account & $\mathbf{1 7}$ \\
\hline
\end{tabular}

\begin{tabular}{|c|l|c|}
\hline Employee No & Duty & Days \\
\hline 12 & Awaiting transfer & $\mathbf{0}$ \\
\hline 13 & 93 personnel files & $\mathbf{1 2}$ \\
\hline 14 & No duty list. Assisting Chief Clerk & $\mathbf{5}$ \\
\hline
\end{tabular}

\begin{tabular}{|c|l|c|}
\hline Employee No & Duty & Days \\
\hline 15 & Telephone, electricity \& water bill settlement & 0.5 \\
\hline & Doctor's overtime \& 1/20 & 5.0 \\
\hline & Annual verification \& goods condemning (Once a Year) & $(20)$ \\
\hline
\end{tabular}




\begin{tabular}{|l|l|r|}
\hline & Maintenance of condemning stores & 2 \\
\hline & Check vouchers of all units & 2 \\
\hline & Telephone & 4 \\
\hline & & $\mathbf{1 3 . 5}$ \\
\hline
\end{tabular}

Out of the 15 analyzed officers only 13 had duty list and two officers without duty lists are awaiting transfers. There were two full time employed officers for the duties of stores and shroff. Maximum workload had for the officer number 11 and it was 17 days monthly work plus 22 days annual work. Officer number 10 had only four days work for a month and 3 officers had less than 10 days work for a month.

The proposed subjects with duties are as below.

\section{Administrative Division}

\begin{tabular}{|c|l|c|}
\hline Subject No & Duty & Time \\
\hline 01 & 93 personnel files (Major staff) & 12 \\
\hline & Overtime \& travelling voucher checking & 3 \\
\hline & & $\mathbf{1 5}$ \\
\hline
\end{tabular}

\begin{tabular}{|c|l|c|}
\hline Subject No & Duty & Time \\
\hline 02 & Preliminary examination of accidents & 0.5 \\
\hline & Maintenance of files on accidents & 1 \\
\hline & General, electrical, photocopy \& AC maintenance & 8 \\
\hline & Telephone & 4 \\
\hline & Telephone, electricity \& water bill settlement & 0.5 \\
\hline & & $\mathbf{1 4}$ \\
\hline
\end{tabular}

\begin{tabular}{|c|l|c|}
\hline Subject No & Duty & Time \\
\hline 03 & 48 personnel files & 5 \\
\hline & Travelling claims (All staff) & 7 \\
\hline & Vehicle fuel supply management & 3 \\
\hline & & $\mathbf{1 5}$ \\
\hline
\end{tabular}

\begin{tabular}{|c|l|c|}
\hline Subject No & Duty & Time \\
\hline 04 & Leave, Train pass, Train warrant & 1.5 \\
\hline & Doctors overtime \& Combined Allowance voucher & 6 \\
\hline & Purchasing of office newspaper & 0.5 \\
\hline
\end{tabular}




\begin{tabular}{|l|l|c|}
\hline & Office tax payments & \\
\hline & Uniform duty & 1 \\
\hline & Office identity card related duties & 0.5 \\
\hline & "Agrahara” claims & 0.5 \\
\hline & Officer allocation for mobile clinics & 1 \\
\hline & Security officers' payments & 1 \\
\hline & Janitorial service payments & 2 \\
\hline & Check vouchers of all units & $\mathbf{1 4}$ \\
\hline
\end{tabular}

\begin{tabular}{|l|l|c|}
\hline Subject No & Duty & Time \\
\hline 05 & Transport subject (Insurance, Repair, Daily records) & 4 \\
\hline & Doctor's overtime \& 1/20 & 5 \\
\hline & Overtime (Except Doctors) & 4 \\
\hline & Casual employee attendance verification for salary & 1 \\
\hline
\end{tabular}

\section{Accounts Division}

\begin{tabular}{|c|c|c|}
\hline Subject No & Duty & Responsibility \\
\hline \multirow[t]{5}{*}{01} & Annual procurement plane & \multirow{5}{*}{$\begin{array}{c}\text { Total procurement subject } \\
\text { responsibility }\end{array}$} \\
\hline & Monthly progress of procurement & \\
\hline & Calling for tenders & \\
\hline & Calling for quotations & \\
\hline & Procurement estimates & \\
\hline Subject No & Duty & Time \\
\hline \multirow[t]{10}{*}{02} & Vote ledger \& reports & 4 \\
\hline & General deposit account & 1 \\
\hline & Salary preparation \& issuing pay slips & 9 \\
\hline & Issuing T-10 & 1 \\
\hline & Carder report & 1 \\
\hline & Preparation of imprest (Liquid Cash) applications & 1 \\
\hline & Preparation of appropriation account & 1 \\
\hline & Annual verification \& goods condemning (Once a year) & $(10)$ \\
\hline & Preparation of annual estimates (once a year) & (11) \\
\hline & & 18 \\
\hline
\end{tabular}

\begin{tabular}{|l|l|c|}
\hline Subject No & Duty & Time \\
\hline
\end{tabular}




\begin{tabular}{|c|l|c|}
\hline 03 & Cask book maintenance & 6 \\
\hline & Maintenance of allocation records & 0.5 \\
\hline & CIGAS/GPS & 5 \\
\hline & Allocation of foreign funds & 2 \\
\hline & Bank reconciliation & 1 \\
\hline & Preparation of expenditure reports (monthly) & 1 \\
\hline & Maintenance of government salary structure & 0.5 \\
\hline & Petty cash balance (School Health Office) & $(10)$ \\
\hline & Annual verification \& goods condemning (Once a year) & $(11)$ \\
\hline & Preparation of annual estimates (once a year) & $\mathbf{1 8}$ \\
\hline
\end{tabular}

\begin{tabular}{|c|l|c|c|}
\hline Subject No & Duty & Time & Availability \\
\hline 04 & Proper maintenance of the stores & \multirow{2}{*}{ Full time stores } \\
\hline & Item estimation \& requesting & & \multirow{2}{*}{} \\
\hline & Maintenance of record books & \\
\hline & Issue of office items & \\
\hline & Issue items for janitorial service & & \\
\hline & Maintenance of condemning stores & 2 & \\
\hline & Surgical store document management & 4 & \\
\hline
\end{tabular}

\begin{tabular}{|l|l|c|c|}
\hline Subject No & Duty & Time & Availability \\
\hline 05 & Shroff & & Full time \\
\hline & Petty cash book & 1 & \\
\hline & Housing loan \& 10-month loan account & 1 & \\
\hline & Salary arrears \& Ledger balancing & 2 & \\
\hline & Internal \& General audit reports & 2 & \\
\hline & & $\mathbf{6}$ & \\
\hline
\end{tabular}

Proposed system has only 10 subjects for the approved posts and duties were distributed scientifically to utilize all the employees equally and efficiently. Similar type of work grouped for the purpose of efficient service delivery and to increase the compliance of the officers. Ten subjects were regrouped to 2 divisions under Accountant and Administrative Officer. Hence basic management principles of chain of command and line of authority will be truly evident through efficiency in future. Five subjects were proposed to each division based on administrative requirements.

\section{CONCLUSION}


International Journal of Scientific and Research Publications, Volume 11, Issue 7, July 2021

Backoffice duties of the FHB are distributed unscientifically and some of the officers had unacceptable quantity of less work to do in the office due to sudden influx of 5 new Public Management Assistants and those on transfer order have not yet released. Therefore, it is important to implement proposed reallocation of duties, which was done following a scientific method. Further, I recommend doing a detailed work study in future to improve the efficiency of the employees.

\section{REFERENCES}

[1] Family Health Bureau (2020) [online]. Available at: http://fhb.health.gov.lk/index.php/en/

[2] Kumara, K. A. J. S. (2020) Key informant Interview, Administrative Officer, Family Health Bureau.

[3] Seenadeera, S.R. (2020) Key informant Interview, Accountant, Family Health Bureau.

[4] Perera, P. I. K. (2020) Key informant Interview, Government Health Management officer, Family Health Bureau.

[5] Gamage, A. G. V. N. (2020) Key informant Interview, Development officer, Family Health Bureau.

\section{AUTHORS}

First Author -

Karunatilaka M. N., MSc (Medical Administration), MB;BS, Registrar in Medical Administration

Second Author - De Silva, C., MD, MB;BS, Director, Family Health Bureau.

Corresponding Author - Karunatilaka M. N., mudith_karunatilaka@yahoo.com, 094714867385 